Contributions to Game Theory and Management, XIV, 38-48

\title{
Non-Zero Sum Network Games with Pairwise Interactions*
}

\author{
Mariia A. Bulgakova \\ St. Petersburg State University, \\ 7/9 Universitetskaya nab., Saint Petersburg, 199034, Russia \\ E-mail: mari_bulgakova@mail.ru
}

\begin{abstract}
In the paper non-zero sum games on networks with pairwise interactions are investigated. The first stage is network formation stage, where players chose their preferable set of neighbours. In all following stages simultaneous non-zero sum game appears between connected players in network. As cooperative solutions the Shapley value and $\tau$-value are considered. Due to a construction of characteristic function both formulas are simplified. It is proved, that the coefficient $\lambda$ in $\tau$-value is independent from network form and number of players or neighbours and is equal to $\frac{1}{2}$. Also it is proved that in this type of games on complete network the Shapley value and $\tau$-value are coincide.
\end{abstract}

Keywords: cooperative games, network games, dynamic games, the Shapley value, $\tau$-value.

\section{Introduction}

The theory of cooperative network games is an important part of modern game theory that will be used to construct solutions in games on networks. This theory includes the cooperative trajectory, the strategies that generate it, the payoff along the cooperative trajectory, as well as the distribution of payoff between the players and the analysis of the dynamic stability of solutions (Petrosyan, Danilov, 1979).

The network illustrates the interaction between players and the possibility of cooperation. The main attention is given to cooperative behavior of player that is behavior in which the joint payoff of players will be maximal.

The principle of pairwise interaction used in this article was first introduced in (Dyer, Mohanaraj, 2011) with the aim of finding a Nash equilibrium in nonzerosum game. This principle implies splitting the game into a family of simultaneous games between pairs of players - the vertices of the same edge in the network. In (Bulgakova, Petrosyan, 2015) the first time the principle of pairwise interaction was applied to cooperative two-stage games. The paper (Bulgakova, Petrosyan, 2019(a)) is devoted to finding the basic solutions of two-stage cooperative games with pairwise interaction and also the supermodularity of the characteristic function is proved. This property significantly increases the value of such a solution as the Shapley value, since in the case of a convex game it always belongs to the core. In this regard, in games based on pairwise interaction, this solution is of particular interest, also because of possibility to simplify the corresponding calculations. Considering the construction of the characteristic function, another solution proposed in (Tijs, 1987), namely, $\tau$-value.

In this paper, a special type of multi-stage cooperative games on the network is considered, which is distinguished by the way of constructing the characteristic function. Like in (Bulgakova, Petrosyan, 2019(b)), first the cooperative trajectory

${ }^{*}$ This work was supported by the Russian Science Foundation (project No.17-11-01079). https://doi.org/10.21638/11701/spbu31.2021.03 
is found, and then the characteristic function is calculated taking into account the cooperative strategies of players. As a solution, the Shapley value and $\tau$-value are considered, for the latter, the coefficient $\lambda$ is calculated, which is the same for any number of players and for any network design in a given game. It is also shown that in this game the Shapley value and $\tau$-value coincide. The illustrative example is presented.

\section{The model}

Let an abstract finite space $\mathbf{Z}$ be given, called the state space. On the first stage in state (position) $z_{0} \in \mathbf{Z}$, players form the network $g\left(z_{0}\right)$, where the vertices are players, and edges are connections between them. In every following state $z_{k} \in \mathbf{Z}$ players can change the network by cancelling connections, and after playing n-person non-zero sum game $\Gamma\left(z_{k}\right)$ on network $g\left(z_{k}\right)$.

Define the rule of network formation $g\left(z_{0}\right)$ on the first stage similar to [8]: in the initial state $z_{0}$ every player $i \in N$ choose his behavior $b_{i}\left(z_{0}\right)=\left(b_{i 1}\left(z_{0}\right), \ldots, b_{i n}\left(z_{0}\right)\right)$ - $n$-dimensional connection vector with components taking values 0 or 1 . Introduce following notations: $M_{i} \subseteq N \backslash i$ - those players, whom player $i \in N$ is offering connection. The value $a_{i} \in\{0, \ldots, n-1\}$ is maximal number of player $i$ connections on every stage. If $M_{i}=N \backslash\{i\}$, player $i$ can offer a connection to all players, and if $a_{i}=n-1$, player $i$ can support any number of possible connections. Thus, each player is restricted by the number of connections $a_{i}$, which he has to offer, and set $M_{i}$ of players, available for creating connections. In other words, on the first stage control $y_{i}\left(z_{0}\right)$ is vector of connection offers $b_{i}\left(z_{0}\right)$.

Player $i$ chose subset of players $Q_{i} \subset M_{i}$, which he want to see as his neighbors.

Then components of the vector $b_{i}\left(z_{0}\right)$ are defined in following way:

$$
b_{i j}\left(z_{0}\right)=\left\{\begin{array}{l}
1, \quad \text { if } j \in Q_{i}, \\
0, \text { if } j \notin Q_{i} \text { or } i=j,
\end{array}\right.
$$

under additional condition

$$
\sum_{j \in N} b_{i j}\left(z_{0}\right) \leq a_{i}
$$

Condition (2) means, that the number of possible connections is limited for each player. Note, that $\left|Q_{i}\right| \leq a_{i}$. The connection can be realized only with a player from the subset $Q_{i}$.

The connection $i j$ is realized if and only if $b_{i j}\left(z_{0}\right)=b_{j i}\left(z_{0}\right)=1$, i. e. $i \in Q_{j}, j \in$ $Q_{i}$. Formed connections $i j$ create edges of the network $g\left(z_{0}\right)$, where players are vertices, i. e., if $b_{i j}=b_{j i}=1$, then in the network $g$ an edge with end vertices $i$ and $j$ appears.

Denote by $N_{i}\left(g\left(z_{0}\right)\right)$ neighbors of player $i$ in the network $g\left(z_{0}\right)$, i. e. $N_{i}\left(g\left(z_{0}\right)\right)=$ $\left\{j \in N \backslash\{i\}: i j \in g\left(z_{0}\right)\right\}$.

After network $g\left(z_{0}\right)$ formation players pass to state $z_{1}\left(g\left(z_{0}\right)\right)$, which depend on network $g\left(z_{0}\right)$. In state $z_{1}\left(g\left(z_{0}\right)\right)$ players are given the opportunity to remove some of previously established connections, thus rebuilding the network $g\left(z_{0}\right)$ in $g\left(z_{1}\right)$ and forming new sets of neighbors $N_{i}\left(g\left(z_{1}\right)\right)$. On the network $g\left(z_{1}\right)$ players play the game $\Gamma\left(z_{1}\right)$, which is a simultaneous non-zero sum game between neighbors on the network.

On the second stage, in the state $z_{1}$ player $i, i=\overline{1, n}$, chooses control $y_{i}\left(z_{1}\right)=$ $\left(b_{i}\left(z_{1}\right), x_{i}\left(z_{1}\right)\right)$ from finite set $Y_{i}$, which, unlike the first stage, contains an additional 
component $x_{i}\left(z_{1}\right)$ - behavior in game $\Gamma\left(z_{1}\right)$. And $b_{i}\left(z_{1}\right)$ is a vector with components 0 or 1 , obtained by the following rule:

$$
b_{i j}\left(z_{1}\right)=\left\{\begin{array}{l}
1, \text { save link } i j, \\
0, \text { delete link } i j,
\end{array}\right.
$$

i. e. the player in the second stage has the ability to delete existing links, however he does not have the ability to create new. $x_{i}\left(z_{1}\right)$ is behavior of player $i$ in game $\Gamma\left(z_{1}\right)$ and it is chosen from finite set $X_{i}\left(z_{1}\right)$, defined in state $z_{1}$.

Let $y\left(z_{1}\right)=\left(y_{1}\left(z_{1}\right), \ldots, y_{n}\left(z_{1}\right)\right)$ be a strategy profile in game $\Gamma\left(z_{1}\right)$. Payoff of player $i$ in game $\Gamma\left(z_{1}\right)$ equals to:

$$
H_{i}\left(z_{1}\right)=\sum_{j \in N_{i}\left(g\left(z_{1}\right)\right)} h_{i}\left(y_{i}\left(z_{1}\right), y_{j}\left(z_{1}\right)\right),
$$

where $g\left(z_{1}\right)$ is network, resulting from the strategy profile $y\left(z_{1}\right)$, which provides the ability to remove some edges from the network $g\left(z_{0}\right)$, the functions $h_{i}\left(x_{i}\left(z_{1}\right), x_{j}\left(z_{1}\right)\right) \geq 0$ are given for all $i \in N$ and for all pairs $i j$, i. e. for all edges of network $g\left(z_{1}\right)$ and all possible states $z \in \mathbf{Z}$.

Suppose that in state $z_{k-1} \in Z$ in game $\Gamma\left(z_{k-1}\right)$ players $i \in N$ chose controls $\left(y_{1}\left(z_{k-1}\right), \ldots, y_{n}\left(z_{k-1}\right)\right)$. As a result of the choice of these controls, the transition in a state $z_{k}$ takes place, where the game $\Gamma\left(z_{k}\right)$ happens, with payoffs $h_{i}\left(x_{j}\left(z_{k}\right), x_{i}\left(z_{k}\right)\right)$. That is, the state at the next stage of the game depends on the state at the current stage and on the controls selected at this stage. We can define the mapping $T: \mathbf{Z} \times Y_{1} \times Y_{2} \times \ldots \times Y_{n} \rightarrow \mathbf{Z}$ by the formula

$$
z_{k}=T\left(z_{k-1} ; y_{1}\left(z_{k-1}\right), y_{2}\left(z_{k-1}\right), \ldots, y_{n}\left(z_{k-1}\right)\right), \quad k=\overline{1, \ell}
$$

The mapping $T$ uniquely identifies the state $z_{k}$, which follows the state $z_{k-1}$, provided that the controls have been selected $y_{1}\left(z_{k-1}\right), y_{2}\left(z_{k-1}\right), \ldots, y_{n}\left(z_{k-1}\right)$ at state $z_{k-1}$.

Consider multistage game $G(z)$, which happens as follows. The game $G\left(z_{0}\right)$ starts in state $z_{0}$. In state $z_{0}$ the network $g\left(z_{0}\right)$ is being formed, after the players pass to the state $z_{1}$. In state $z_{k-1}, k=\overline{1, \ell-1}$ players choose controls $y_{1}\left(z_{k-1}\right), y_{2}\left(z_{k-1}\right), \ldots, y_{n}\left(z_{k-1}\right)$, play game $\Gamma\left(z_{k-1}\right)$ and pass to the state $z_{k}=T\left(z_{k-1} ; y_{1}\left(z_{k-1}\right), y_{2}\left(z_{k-1}\right), \ldots, y_{n}\left(z_{k-1}\right)\right)$. Game ends on stage $\ell+1$ in state $z_{\ell}$. Thus, as a result of control choices at each stage of the game, the path $z_{0}, z_{1}, \ldots, z_{k}, \ldots, z_{\ell}$ is realised.

State $z$ is called admissible, if there is a sequence of controls and a sequence of states generated by these controls $z_{0}, z_{1}, \ldots z_{k}, \quad k \leq \ell$, such that $z_{k}=z$.

The concept of a strategy in the resulting multistage game is introduced in a natural way: $y_{i}(\cdot), i \in N$, - as a rule, which to each admissible state $z$ of game matches components $b_{i}(z), x_{i}(z)$ controls in this state, i. e. selection of links to remove and selection of behavior $x_{i}(z)$ in game $\Gamma(z)$. From the above description it follows that any strategy profile $y(\cdot)=\left\{y_{1}(\cdot), \ldots, y_{n}(\cdot)\right\}$ uniquely determines the path in the game, and, consequently, the payoff of each player as the sum of his payoffs in games realized along the path:

$$
H_{i}(y(\cdot))=\sum_{k=1}^{\ell} \sum_{j \in N_{i}(g(z))} h_{i}\left(y_{i}\left(z_{k}\right), y_{j}\left(z_{k}\right)\right) .
$$


Note that the set of all possible paths in the multistage game $G(z)$ is finite, and thus the set of all admissible states in the game is finite. We denote this set by $\overline{\mathbf{Z}} \subset \mathbf{Z}$.

Suppose players choose controls $\bar{y}_{i}(z), i \in N$, which maximize their joint payoff in the game $G(z)$, i. e.

$$
\sum_{k=1}^{\ell} \sum_{i \in N} H_{i}\left(\bar{y}_{1}\left(z_{k}\right), \ldots, \bar{y}_{n}\left(z_{k}\right)\right)=\max _{y} \sum_{k=1}^{\ell} \sum_{i \in N} H_{i}\left(y_{1}\left(z_{k}\right), \ldots, y_{n}\left(z_{k}\right)\right) .
$$

Strategy profile $\bar{y}=\left(\bar{y}_{1}, \ldots, \bar{y}_{n}\right)$ will be called the cooperative behavior in the game $G(z)$, and trajectory $\left(\bar{z}_{0}, \bar{z}_{1}, \ldots, \bar{z}_{\ell}\right)$ which corresponds to the controls $\bar{y}_{i}(z), i \in N$, - we will call cooperative trajectory $\left(z_{0}=\bar{z}_{0}\right)$.

Consider single stage game $\Gamma(z)$ in arbitrary state $z \in \mathbf{Z}$ in a cooperative form and define its characteristic function $v(S ; z), S \subset N$, for every subset (coalition) $S \subset N$ according to the rule:

$$
\begin{aligned}
& v(\emptyset ; z)=0, \\
& v(\{i\} ; z)=0, \\
& v(\{i j\} ; z)=\left\{\begin{array}{cr}
h_{i}\left(\bar{x}_{i}(z) ; \bar{x}_{j}(z)\right)+h_{j}\left(\bar{x}_{j}(z) ; \bar{x}_{i}(z)\right), & \text { if } j \in N_{i}(g(z)), \\
0, & \text { in other case }
\end{array}\right. \\
& v(S ; z)=\sum_{i \in S} \sum_{j \in N_{i}(g(z)) \cap S} h_{i}\left(\bar{x}_{i}(z) ; \bar{x}_{j}(z)\right), \\
& v(N ; z)=\sum_{i \in N} \sum_{j \in N_{i}(g(z))} h_{i}\left(\bar{x}_{i}(z) ; \bar{x}_{j}(z)\right),
\end{aligned}
$$

where $\bar{x}_{i}(z), \bar{x}_{j}(z)$ are obtained according (5).

Earlier it was proved, that the characteristic function $v(S ; z)$ is supermodular and corresponding game is convex (see Bulgakova, Petrosyan, 2019(a)).

Here, unlike the previous approaches, in which the characteristic function was constructed as the maximin (lower) value of the game between the coalition $S$ and additional coalition $N \backslash S$, the process of construction of this function is carried out as follows. To calculate the value of the characteristic function, it is necessary to determine the cooperative behavior in the game $G\left(z_{0}\right)$ and then calculate $v\left(S ; z_{k}\right), k=\overline{1, \ell}$, under the assumption that players choose cooperative behavior as a control component.

Find the characteristic function $V\left(S ; z_{k}\right)$ of multistage game $G\left(z_{k}\right)$, started in position $z_{k}$, as the sum of the payoffs of the coalitions $S$ along the cooperative trajectory $\left(\bar{y}\left(z_{0}\right), \bar{y}\left(z_{1}\right), \ldots, \bar{y}\left(z_{l}\right)\right)$ in $\ell-k+1$ stages, starting from $k$ :

$$
\begin{gathered}
V\left(S ; z_{k}\right)=\sum_{r=k}^{\ell} v\left(S ; z_{r}\right)=\sum_{r=k}^{\ell} \sum_{i \in S} \sum_{j \in N_{i}\left(g\left(z_{r}\right)\right) \cap S} h_{i}\left(\bar{x}_{i}\left(z_{r}\right), \bar{x}_{j}\left(z_{r}\right)\right), \\
V\left(S ; z_{\ell}\right)=v\left(S ; z_{\ell}\right) .
\end{gathered}
$$




\section{The $\tau$-value}

Consider as a solution for game $G\left(z_{0}\right)$ the $\tau$-value (Tijs, 1987). Start with a special case - single stage game $\Gamma\left(z_{1}\right)$ which appears after network formation stage.

Proposition 1. In the game $\Gamma\left(z_{1}\right)$, $\tau$-value equals to:

$$
\begin{gathered}
\tau_{j}\left(N, v, z_{1}\right)=\frac{1}{2}\left(v\left(N, z_{1}\right)-v(N \backslash\{j\}), z_{1}\right)= \\
=\frac{1}{2} \sum_{i \in N_{j}\left(g\left(z_{1}\right)\right)}\left(h_{i}\left(\bar{x}_{i}\left(z_{1}\right), \bar{x}_{j}\left(z_{1}\right)\right)+h_{j}\left(\bar{x}_{i}\left(z_{1}\right), \bar{x}_{j}\left(z_{1}\right)\right)\right) .
\end{gathered}
$$

Proof. We use values of characteristic function for a single player and maximal coalition $N(6),(10)$ :

Components of $\tau$-value for convex game can be calculate by the formula:

$$
\tau_{j}(N, v)=\lambda(v(N)-v(N \backslash\{j\}))+(1-\lambda) v(\{j\})
$$

where coefficients $\lambda$ is defined from equation

$$
\sum_{j \in N}(\lambda((v(N)-v(N \backslash\{j\}))+(1-\lambda) v(\{j\}))=v(N)
$$

Since $v(\{j\})=0$ (see $(7))$, the term $(1-\lambda) v(\{i\})$ also is equal to zero.

Therefore, for two-stage game with pairwise interactions we have following formula for calculating $\tau$-value:

$$
\tau_{i}\left(N, v, z_{1}\right)=\lambda\left(v\left(N, z_{1}\right)-v(N \backslash\{i\}), z_{1}\right)
$$

where coefficients $\lambda$ is defined from

$$
\sum_{j \in N} \lambda\left(v\left(\left(N, z_{1}\right)-v(N \backslash\{j\}), z_{1}\right)=v\left(N, z_{1}\right)\right.
$$

Calculate the values of the difference $v\left(N, z_{1}\right)-v\left(N \backslash\{j\}, z_{1}\right)$. If player $j$ does not belong to coalition $N, N$ has no reason to support connections with $j$. Thus $N$ loses payoff on these connections. So the considered difference will be equal:

$$
v\left(N, z_{1}\right)-v\left(N \backslash\{j\}, z_{1}\right)=\sum_{i \in N_{j}\left(g\left(z_{1}\right)\right)}\left(h_{i}\left(\bar{x}_{i}\left(z_{1}\right), \bar{x}_{j}\left(z_{1}\right)\right)+h_{j}\left(\bar{x}_{i}\left(z_{1}\right), \bar{x}_{j}\left(z_{1}\right)\right)\right) .
$$

Now go back to the equation (12), and substitute into it the values of the characteristic function.

$$
\begin{gathered}
\sum_{j \in N} \lambda\left(\sum_{i \in N_{j}\left(g\left(z_{1}\right)\right)}\left(h_{i}\left(\bar{x}_{i}\left(z_{1}\right), \bar{x}_{j}\left(z_{1}\right)\right)+h_{j}\left(\bar{x}_{i}\left(z_{1}\right), \bar{x}_{j}\left(z_{1}\right)\right)\right)\right)= \\
=\sum_{j \in N} \sum_{i \in N_{j}\left(g\left(z_{1}\right)\right)} h_{i}\left(\bar{x}_{i}\left(z_{1}\right) ; \bar{x}_{j}\left(z_{1}\right)\right)
\end{gathered}
$$


We get

$$
\begin{gathered}
\lambda \sum_{j \in N}\left(\sum_{i \in N_{j}\left(g\left(z_{1}\right)\right)}\left(h_{i}\left(\bar{x}_{i}\left(z_{1}\right), \bar{x}_{j}\left(z_{1}\right)\right)+h_{j}\left(\bar{x}_{i}\left(z_{1}\right), \bar{x}_{j}\left(z_{1}\right)\right)\right)\right)= \\
=\sum_{j \in N} \sum_{i \in N_{j}\left(g\left(z_{1}\right)\right)} h_{i}\left(\bar{x}_{i}\left(z_{1}\right) ; \bar{x}_{j}\left(z_{1}\right)\right)
\end{gathered}
$$

Due to double summation on the left side of equality $\left(h_{i}\left(\bar{x}_{i}\left(z_{1}\right), \bar{x}_{j}\left(z_{1}\right)\right)+h_{j}\left(\bar{x}_{i}\left(z_{1}\right), \bar{x}_{j}\left(z_{1}\right)\right)\right.$, and since the summation is carried out over $i$ and $j$, we get:

$$
\lambda \cdot 2 v\left(N, z_{1}\right)=v\left(N, z_{1}\right)
$$

Cancelling the same multipliers on the right and left sides of the equation, we get:

$$
\lambda=\frac{1}{2}
$$

Thus, in cooperative network game $\Gamma\left(z_{1}\right)$ with pairwise interactions, , coefficient $\lambda$ in $\tau$-value is equal to $\frac{1}{2}$, and does not depend on the number of players, or on the number of connections between them, or on the structure of the network. And we get that

$$
\tau_{j}\left(N, v, z_{1}\right)=\frac{1}{2} \sum_{i \in N_{j}\left(g\left(z_{1}\right)\right)}\left(h_{i}\left(\bar{x}_{i}\left(z_{1}\right), \bar{x}_{j}\left(z_{1}\right)\right)+h_{j}\left(\bar{x}_{i}\left(z_{1}\right), \bar{x}_{j}\left(z_{1}\right)\right)\right) .
$$

Proposition is proved.

We can apply this formula for arbitrary stage $k$, because of components of the $\tau$ value depend only from the payoffs on this stage.

Now we can find the $\tau$-value for multistage game $G\left(z_{k}\right)$, starting from arbitrary position $z_{k}$

$$
\tau_{j}^{\prime}\left(N, v, z_{0}\right)=\frac{1}{2} \sum_{r=k}^{\ell} \sum_{i \in N_{j}\left(g\left(z_{k}\right)\right)}\left(h_{i}\left(\bar{x}_{i}\left(z_{k}\right), \bar{x}_{j}\left(z_{k}\right)\right)+h_{j}\left(\bar{x}_{i}\left(z_{k}\right), \bar{x}_{j}\left(z_{k}\right)\right)\right) .
$$

\section{The Shapley value}

Now consider another solution, the Shapley value, and compare it with previous on the special network - complete network.

Proposition 2. In multistage network game $G\left(z_{0}\right)$ with pairwise interactions on the complete network $\tau$-value and the Shapley value coincide.

Proof. Earlier, we obtained a simplified formula for the components of the Shapley value for a two-stage cooperative game with pairwise interaction on complete network:

$$
\varphi_{i}[v]=\frac{1}{2} \sum_{j \in N} m_{i j}
$$

where $m_{i j}$ is maximal joint payoff for pair $i j$ which create an edge in network.

Now consider how we can simplify formula for calculating components of the Shapley value in considered game. As previous, we start from single stage game $\Gamma\left(z_{0}\right)$ and then generalize the result to multistage game. 
For components of the Shapley value we will use following formula (Shapley, 1953):

$$
\varphi_{j}[v]=\sum_{T \mid j \in T \subset N} \frac{(t-1) !(n-t) !}{n !}[v(T)-v(T \backslash j)]
$$

Calculate the difference in values of characteristic functions, $v(T)-v(T \backslash i)$, when $T=N$. For arbitrary coalition $T$ we will have:

$$
v\left(T, z_{1}\right)-v\left(T \backslash\{j\}, z_{1}\right)=\sum_{i \in N_{j}\left(g\left(z_{1}\right)\right) \cap T}\left(h_{i}\left(\bar{x}_{i}\left(z_{1}\right), \bar{x}_{j}\left(z_{1}\right)\right)+h_{j}\left(\bar{x}_{i}\left(z_{1}\right), \bar{x}_{j}\left(z_{1}\right)\right)\right) .
$$

Note, that in (19), in the term with a fixed value $(t)$, the sum $\left(h_{i}\left(\bar{x}_{i}\left(z_{1}\right), \bar{x}_{j}\left(z_{1}\right)\right)+\right.$ $\left.h_{j}\left(\bar{x}_{i}\left(z_{1}\right), \bar{x}_{j}\left(z_{1}\right)\right)\right)$ will occur exactly $C_{n-2}^{t-2}$ times.

As a result we have:

$$
\begin{gathered}
\varphi_{j}[v]= \\
=\sum_{T \mid j \in T \subset N} \frac{(t-1) !(n-t) !}{n !} \cdot C_{n-2}^{t-2} \cdot \sum_{i \in N_{j}\left(g\left(z_{1}\right)\right) \cap T}\left(h_{i}\left(\bar{x}_{i}\left(z_{1}\right), \bar{x}_{j}\left(z_{1}\right)\right)+h_{j}\left(\bar{x}_{i}\left(z_{1}\right), \bar{x}_{j}\left(z_{1}\right)\right)\right) .
\end{gathered}
$$

And, simplifying, we get:

$$
\begin{gathered}
\varphi_{j}[v]=\sum_{i \in N_{j}\left(g\left(z_{1}\right)\right) \cap T}\left(h_{i}\left(\bar{x}_{i}\left(z_{1}\right), \bar{x}_{j}\left(z_{1}\right)\right)+h_{j}\left(\bar{x}_{i}\left(z_{1}\right), \bar{x}_{j}\left(z_{1}\right)\right)\right) \cdot \sum_{t=2}^{n} \frac{t-1}{n \cdot(n-1)}(21) \\
\varphi_{j}[v]=\sum_{i \in N_{j}\left(g\left(z_{1}\right)\right) \cap T}\left(h_{i}\left(\bar{x}_{i}\left(z_{1}\right), \bar{x}_{j}\left(z_{1}\right)\right)+h_{j}\left(\bar{x}_{i}\left(z_{1}\right), \bar{x}_{j}\left(z_{1}\right)\right)\right) \cdot\left(\frac{1}{n \cdot(n-1)}+\frac{1}{n}\right) \cdot \frac{(n-1)}{2}
\end{gathered}
$$

Finally we have:

$$
\varphi_{j}[v]=\frac{1}{2} \cdot \sum_{i \in N_{j}\left(g\left(z_{1}\right)\right) \cap T}\left(h_{i}\left(\bar{x}_{i}\left(z_{1}\right), \bar{x}_{j}\left(z_{1}\right)\right)+h_{j}\left(\bar{x}_{i}\left(z_{1}\right), \bar{x}_{j}\left(z_{1}\right)\right)\right)
$$

Thus $\tau$-value coincides with the Shapley value in game $\Gamma\left(z_{1}\right)$ on complete network. In multistage game $G\left(z_{0}\right)$ we have following formula for components of the Shapley value:

$$
\varphi_{j}[v]=\frac{1}{2} \cdot \sum_{r=k}^{\ell} \sum_{i \in N_{j}\left(g\left(z_{0}\right)\right) \cap T}\left(h_{i}\left(\bar{x}_{i}\left(z_{0}\right), \bar{x}_{j}\left(z_{0}\right)\right)+h_{j}\left(\bar{x}_{i}\left(z_{0}\right), \bar{x}_{j}\left(z_{0}\right)\right)\right)
$$

which also coincides with $\tau$-value on complete network.

\section{Example}

Consider the case, where $N=3, \ell=3$, i. e. the game consists of four stages and starts in the state $z_{0}$. In this state set $M_{i}$ of players, whom player $i$ can offer a connection, is given

$$
M_{1}=\{2,3\}, \quad M_{2}=\{1,3\}, \quad M_{3}=\{1,2\},
$$


as well as restrictions on the number of connections that each player can support:

$$
a_{1}=1, \quad a_{2}=1, \quad a_{3}=2
$$

In state $z_{0}$ players choose behaviors $b_{i}\left(z_{0}\right)$ - formed a network $g\left(z_{0}\right)$ and then pass to the state $z_{1}$. In every state $z_{k}, k \geq 1$ players choose controls $y_{i}\left(z_{k}\right)=$ $\left(b_{i}\left(z_{k}\right), x_{i}\left(z_{k}\right)\right)$, where $b_{i}\left(z_{k}\right)$ - vector regulating player connections (with components 1 or 0$)$, and $x_{i}\left(z_{k}\right)$ is defined as

$$
\begin{gathered}
x_{1}\left(z_{k}\right)=x_{1}(z) \in X_{1}=\left\{x_{1}^{1}(z), x_{1}^{2}(z)\right\} \\
x_{2}\left(z_{k}\right)=x_{2}(z) \in X_{2}=\left\{x_{2}^{1}(z), x_{2}^{2}(z)\right\}, \quad x_{3}\left(z_{k}\right)=x_{3}(z) \in X_{3}\left\{x_{3}^{1}(z), x_{3}^{2}(z)\right\}
\end{gathered}
$$

Thus every player $i$ has a given set of control components $X_{i}$ in all states $z_{k}$.

For admissible states $z_{k}, \quad k \geq 1$, and all possible strategies payoffs are given $h_{i}\left(x_{i}\left(z_{r}\right), x_{j}\left(z_{r}\right)\right)$ in following way: $h_{i}\left(x_{i}\left(z_{r}\right), x_{j}\left(z_{r}\right)\right)$ and $h_{i}^{\prime}\left(x_{i}\left(z_{r}\right), x_{j}\left(z_{r}\right)\right)$.

In state $z_{1}$ the game happens with payoffs $h\left(x_{i}\left(z_{1}\right), x_{j}\left(z_{1}\right)\right)$. In state $z_{1}$ every player $i \in N$ chose his control component $x_{i}\left(z_{1}\right)$, and if all $x_{i}\left(z_{1}\right)=x_{i}^{1}\left(z_{1}\right), i \in N$, then players pass to a state $z_{2}$, in which the game is played with the same payoffs $h_{i}\left(x_{i}\left(z_{2}\right), x_{j}\left(z_{2}\right)\right)$. If at least one of the components $x_{i}\left(z_{1}\right)=x_{i}^{2}\left(z_{1}\right), i \in N$, then in state $z_{2}$ players play a game with payoffs $h_{i}^{\prime}\left(x_{i}\left(z_{2}\right), x_{j}\left(z_{2}\right)\right)$. In a similar way, the transition to the state $z_{3}$ : if all $x_{i}\left(z_{1}\right)=x_{i}^{1}\left(z_{1}\right), i \in N$, then the players in $z_{3}$ use payoffs $h_{i}\left(x_{i}\left(z_{3}\right), x_{j}\left(z_{3}\right)\right)$, again if at least one of the components $x_{i}\left(z_{1}\right)=$ $x_{i}^{2}\left(z_{1}\right), i \in N,-$ payoffs are $h_{i}^{\prime}\left(x_{i}\left(z_{3}\right), x_{j}\left(z_{3}\right)\right)$.

Payoffs $h_{i}\left(x_{i}\left(z_{r}\right), x_{j}\left(z_{r}\right)\right)$ :

$$
\begin{aligned}
& h_{1}\left(x_{1}^{1}, x_{2}^{1}\right)=4, h_{1}\left(x_{1}^{1}, x_{3}^{1}\right)=5, h_{2}\left(x_{2}^{1}, x_{3}^{1}\right)=5, \\
& h_{1}\left(x_{1}^{2}, x_{2}^{1}\right)=3, h_{1}\left(x_{1}^{2}, x_{3}^{1}\right)=3, h_{2}\left(x_{2}^{2}, x_{3}^{1}\right)=1, \\
& h_{1}\left(x_{1}^{1}, x_{2}^{2}\right)=5, h_{1}\left(x_{1}^{1}, x_{3}^{2}\right)=1, h_{2}\left(x_{2}^{1}, x_{3}^{2}\right)=4, \\
& h_{1}\left(x_{1}^{2}, x_{2}^{2}\right)=5, h_{1}\left(x_{1}^{2}, x_{3}^{2}\right)=2, h_{2}\left(x_{2}^{2}, x_{3}^{2}\right)=1, \\
& h_{2}\left(x_{2}^{1}, x_{1}^{1}\right)=4, h_{3}\left(x_{3}^{1}, x_{1}^{1}\right)=5, h_{3}\left(x_{3}^{1}, x_{2}^{1}\right)=5, \\
& h_{2}\left(x_{2}^{1}, x_{1}^{2}\right)=3, h_{3}\left(x_{3}^{1}, x_{1}^{2}\right)=3, h_{3}\left(x_{3}^{1}, x_{2}^{2}\right)=1, \\
& h_{2}\left(x_{2}^{2}, x_{1}^{1}\right)=5, h_{3}\left(x_{3}^{2}, x_{1}^{1}\right)=1, h_{3}\left(x_{3}^{2}, x_{2}^{1}\right)=4, \\
& h_{2}\left(x_{2}^{2}, x_{1}^{2}\right)=5, h_{3}\left(x_{3}^{2}, x_{1}^{2}\right)=2, h_{3}\left(x_{3}^{2}, x_{2}^{2}\right)=1 ;
\end{aligned}
$$

payoffs $h_{i}^{\prime}\left(x_{i}\left(z_{r}\right), x_{j}\left(z_{r}\right)\right)$ :

$$
\begin{aligned}
& h_{1}^{\prime}\left(x_{1}^{1}, x_{2}^{1}\right)=8, h_{1}^{\prime}\left(x_{1}^{1}, x_{3}^{1}\right)=6, h_{2}^{\prime}\left(x_{2}^{1}, x_{3}^{1}\right)=12, \\
& h_{2}^{\prime}\left(x_{1}^{2}, x_{2}^{1}\right)=3, h_{1}^{\prime}\left(x_{1}^{2}, x_{3}^{1}\right)=5, h_{2}^{\prime}\left(x_{2}^{2}, x_{3}^{1}\right)=10, \\
& h_{1}^{\prime}\left(x_{1}^{1}, x_{2}^{2}\right)=7, h_{1}^{\prime}\left(x_{1}^{1}, x_{3}^{2}\right)=4, h_{2}^{\prime}\left(x_{2}^{1}, x_{3}^{2}\right)=5, \\
& h_{1}^{\prime}\left(x_{1}^{2}, x_{2}^{2}\right)=4, h_{1}^{\prime}\left(x_{1}^{2}, x_{3}^{2}\right)=3, h_{2}^{\prime}\left(x_{2}^{2}, x_{3}^{2}\right)=4, \\
& h_{2}^{\prime}\left(x_{2}^{1}, x_{1}^{1}\right)=8, h_{3}^{\prime}\left(x_{3}^{1}, x_{1}^{1}\right)=6, h_{3}^{\prime}\left(x_{3}^{1}, x_{2}^{1}\right)=12, \\
& h_{2}^{\prime}\left(x_{2}^{1}, x_{1}^{2}\right)=3, h_{3}^{\prime}\left(x_{3}^{1}, x_{1}^{2}\right)=5, h_{3}^{\prime}\left(x_{3}^{1}, x_{2}^{2}\right)=10, \\
& h_{2}^{\prime}\left(x_{2}^{2}, x_{1}^{1}\right)=7, h_{3}^{\prime}\left(x_{3}^{2}, x_{1}^{1}\right)=4, h_{3}^{\prime}\left(x_{3}^{2}, x_{2}^{1}\right)=5, \\
& h_{2}^{\prime}\left(x_{2}^{2}, x_{1}^{2}\right)=4, h_{3}^{\prime}\left(x_{3}^{2}, x_{1}^{2}\right)=3, h_{3}^{\prime}\left(x_{3}^{2}, x_{2}^{2}\right)=4 .
\end{aligned}
$$

In state $z_{0}$ players choose their behaviors in order to maximize the total payoff of all players:

$$
b_{1}\left(z_{0}\right)=(0,0,1), \quad b_{2}\left(z_{0}\right)=(0,0,1), \quad b_{3}\left(z_{0}\right)=(1,1,0)
$$

As a result, a network (see fig. 1) is formed. 


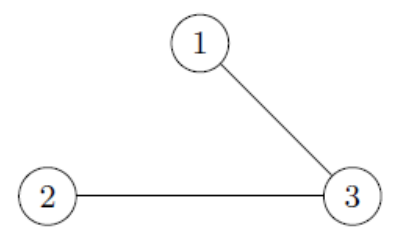

Fig. 1. Network on the first stage of game.

To maximize the joint payoff, it is beneficial for the players to keep connections with all neighbors throughout the game, i. e. $b_{i}\left(z_{0}\right)=b_{i}\left(z_{1}\right)=b_{i}\left(z_{2}\right)=b_{i}\left(z_{3}\right)$, for all $i \in N$. Components of the player controls $\bar{y}_{i}(z)$

$$
\begin{array}{lll}
\bar{x}_{1}\left(z_{1}\right)=x_{1}^{2}, & \bar{x}_{2}\left(z_{1}\right)=x_{2}^{1}, & \bar{x}_{3}\left(z_{1}\right)=x_{3}^{1}, \\
\bar{x}_{1}\left(z_{2}\right)=x_{1}^{1}, & \bar{x}_{2}\left(z_{2}\right)=x_{2}^{2}, & \bar{x}_{3}\left(z_{2}\right)=x_{3}^{1}, \\
\bar{x}_{1}\left(z_{3}\right)=x_{1}^{1}, & \bar{x}_{2}\left(z_{3}\right)=x_{2}^{2}, & \bar{x}_{3}\left(z_{3}\right)=x_{3}^{1} .
\end{array}
$$

Calculate the values of characteristic function $v(S ; z)$ in all states along cooperative trajectory, except $z_{0}$, because the first stage is only network formation stage and players do not receive payoffs:

\begin{tabular}{|c|c|c|c|c|c|c|c|}
\hline$S$ & $\{1\}$ & $\{2\}$ & $\{3\}$ & $\{12\}$ & $\{13\}$ & $\{23\}$ & $\{123\}$ \\
\hline$v\left(S ; \bar{z}_{1}\right)$ & 0 & 0 & 0 & 6 & 6 & 10 & 16 \\
\hline$v\left(S ; \bar{z}_{2}\right)$ & 0 & 0 & 0 & 14 & 12 & 20 & 32 \\
\hline$v\left(S ; \bar{z}_{3}\right)$ & 0 & 0 & 0 & 14 & 12 & 20 & 32 \\
\hline
\end{tabular}

Table 1. The values of characteristic function $v(S ; z)$.

In state $z_{1}$ players choose their controls and, depended on this, move to a new state. In each state, the players have only two alternatives: either, as a result of the choice of controls, they will play the game $\Gamma\left(z_{k}\right)$ with payoffs $h_{i}\left(\bar{x}_{i}\left(z_{k}\right), \bar{x}_{j}\left(z_{k}\right)\right)$ in the next state, or pass in state, where the game will take place with payoffs $h_{i}^{\prime}\left(\bar{x}_{i}\left(z_{k}\right), \bar{x}_{j}\left(z_{k}\right)\right)$.

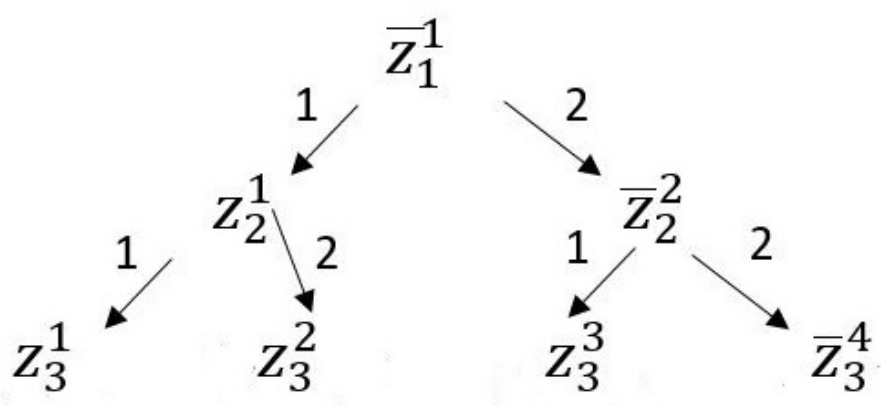

Fig. 2. Tree of all possible states of game.

Numbers 1 and 2 above arrows (fig. 12) show, what payoffs will be used by players in the next state: 1 means $h_{i}\left(\bar{x}_{i}\left(z_{k}\right), \bar{x}_{j}\left(z_{k}\right)\right), 2$ means $h_{i}^{\prime}\left(\bar{x}_{i}\left(z_{k}\right), \bar{x}_{j}\left(z_{k}\right)\right)$. 
Optimal trajectory in game $G\left(z_{0}\right): \bar{z}=\left(z_{0}, z_{1}^{1}, z_{2}^{2}, z_{3}^{4}\right)=\left(\bar{z}_{0}, \bar{z}_{1}, \bar{z}_{2}, \bar{z}_{3}\right)$. Calculate the values of characteristic function in multistage game $G\left(z_{0}\right)$ :

\begin{tabular}{|c|c|c|c|c|c|c|c|}
\hline$S$ & $\{1\}$ & $\{2\}$ & $\{3\}$ & $\{12\}$ & $\{13\}$ & $\{23\}$ & $\{123\}$ \\
\hline$V\left(S ; \bar{z}_{3}\right)$ & 0 & 0 & 0 & 14 & 12 & 20 & 32 \\
\hline$V\left(S ; \bar{z}_{2}\right)$ & 0 & 0 & 0 & 28 & 24 & 40 & 64 \\
\hline$V\left(S ; \bar{z}_{1}\right)$ & 0 & 0 & 0 & 34 & 30 & 50 & 80 \\
\hline
\end{tabular}

Table 2. The values of characteristic function in multistage game $G\left(z_{0}\right)$.

Calculate $\tau$-value and the Shapley value in every stage

$$
\begin{gathered}
\tau_{1}\left(N, v, z_{1}\right)=\frac{1}{2}\left(h_{1}\left(\bar{x}_{1}^{2}, \bar{x}_{2}^{1}\right)+h_{2}\left(\bar{x}_{2}^{1}, \bar{x}_{1}^{2}\right)+h_{1}\left(\bar{x}_{1}^{2}, \bar{x}_{3}^{1}\right)+h_{3}\left(\bar{x}_{3}^{1}, \bar{x}_{1}^{2}\right)\right)=\frac{1}{2}(0+0+3+3)=3 \\
\tau_{2}\left(N, v, z_{1}\right)=\frac{1}{2}\left(h_{2}\left(\bar{x}_{2}^{1}, \bar{x}_{1}^{2}\right)+h_{1}\left(\bar{x}_{1}^{2}, \bar{x}_{2}^{1}\right)+h_{2}\left(\bar{x}_{2}^{1}, \bar{x}_{3}^{1}\right)+h_{3}\left(\bar{x}_{3}^{1}, \bar{x}_{2}^{1}\right)\right)=\frac{1}{2}(0+0+5+5)=5 \\
\tau_{3}\left(N, v, z_{1}\right)=\frac{1}{2}\left(h_{3}\left(\bar{x}_{3}^{1}, \bar{x}_{1}^{2}\right)+h_{1}\left(\bar{x}_{1}^{2}, \bar{x}_{3}^{1}\right)+h_{3}\left(\bar{x}_{3}^{1}, \bar{x}_{2}^{1}\right)+h_{2}\left(\bar{x}_{2}^{1}, \bar{x}_{3}^{1}\right)\right)=\frac{1}{2}(3+3+5+5)=8 \\
\tau_{1}\left(N, v, z_{2}\right)=\frac{1}{2}\left(h_{1}^{\prime}\left(\bar{x}_{1}^{1}, \bar{x}_{2}^{2}\right)+h_{2}^{\prime}\left(\bar{x}_{2}^{2}, \bar{x}_{1}^{1}\right)+h_{1}^{\prime}\left(\bar{x}_{1}^{1}, \bar{x}_{3}^{1}\right)+h_{3}^{\prime}\left(\bar{x}_{3}^{1}, \bar{x}_{1}^{1}\right)\right)=\frac{1}{2}(0+0+6+6)=6 \\
\tau_{2}\left(N, v, z_{2}\right)=\frac{1}{2}\left(h_{2}^{\prime}\left(\bar{x}_{2}^{2}, \bar{x}_{1}^{1}\right)+h_{1}^{\prime}\left(\bar{x}_{1}^{1}, \bar{x}_{2}^{2}\right)+h_{2}^{\prime}\left(\bar{x}_{2}^{2}, \bar{x}_{3}^{1}\right)+h_{3}^{\prime}\left(\bar{x}_{3}^{1}, \bar{x}_{2}^{2}\right)\right)= \\
=\frac{1}{2}(0+0+10+10)=10 \\
\tau_{3}\left(N, v, z_{2}\right)=\frac{1}{2}\left(h_{3}^{\prime}\left(\bar{x}_{3}^{1}, \bar{x}_{1}^{1}\right)+h_{1}^{\prime}\left(\bar{x}_{1}^{1}, \bar{x}_{3}^{1}\right)+h_{3}^{\prime}\left(\bar{x}_{3}^{1}, \bar{x}_{2}^{2}\right)+h_{2}^{\prime}\left(\bar{x}_{2}^{2}, \bar{x}_{3}^{1}\right)\right)= \\
=\frac{1}{2}(6+6+10+10)=16
\end{gathered}
$$

Components for $\tau_{i}\left(N, v, z_{3}\right)$ are equal to $\tau_{i}\left(N, v, z_{2}\right)$ because the payoffs are the same on these stages. In multistage game $G\left(z_{0}\right)$

$$
\begin{gathered}
\tau_{1}^{\prime}\left(N, v, z_{0}\right)=3+6+6=15 \\
\tau_{2}^{\prime}\left(N, v, z_{0}\right)=5+10+10=25 \\
\tau_{3}^{\prime}\left(N, v, z_{0}\right)=8+16+16=40
\end{gathered}
$$

And the Shapley value coincide with $\tau$-value.

\section{Conclusion}

We considered a special type of multi-stage cooperative games on the network which is distinguished by the way of constructing the characteristic function. The main point here is, finding the cooperative trajectory firstly, and then the characteristic function is calculated taking into account the cooperative strategies of players. As a solution, the Shapley value and $\tau$-value are considered, for the latter, the coefficient $\lambda$ is calculated, which is the same for any number of players and for any network design in a given game. It is also shown that in this game the Shapley value and $\tau$-value coincide. 
Acknowledgements. We acknowledge for the support Russian science foundation (project No.17-11-01079).

\section{References}

Tijs, S.H. (1987). An axiomatization of the ô-value. Mathematical Social Sciences, 13, $177-181$.

Dyer, M. and V. Mohanaraj (2011). Pairwise-Interaction Games. ICALP: Automata, Languages and Programming, P. 159-170.

Shapley, L.S. (1953). A value for n-person games. Contributions to the theory of games II, ed. by H. W. Kuhn, A. W. Tucker. Princeton. P. 307-317.

Bulgakova, M. A. and L. A. Petrosyan (2015). Cooperative network games with pairwise interactions. Mathematical game theory and applications, 4(7), 7-18.

Bulgakova, M. A. (2019a) Solutions of network games with pairwise interactions. Vestnik of Saint-Petersburg State university. Series 10. Applied mathematics. Informatics. Control processes, 15(1), 147-156.

Bulgakova, M. A. (2019b). About one multistage non-zero sum game on the network. Vestnik of Saint-Petersburg State university. Series 10. Applied mathematics. Informatics. Control processes, 15(4), 603-615.

Petrosyan, L. A. and N. N. Danilov (1979). Stability of solutions of non-zero-sum game with transferable payoffs. Vestnik of the Leningrad University. Series 1. Mathematics. Mechanicks. Astronomy, 1, 52-59. 